Mirror mechanism The mechanism that unifies perception and action, transforming sensory representations of the behaviour of others into motor representations of the same behaviour in the observer's brain.

Motor act A movement with a specific motor goal (for example, reaching, grasping and manipulating). The successful achievement of its goal represents the reinforcement of the motor act.

* University of Parma, Department of Neuroscience, and the Italian Institute of Technology, Via Volturno 39, 1-43100 Parma, Italy. ${ }^{\ddagger}$ University of Milan, Department of Philosophy, Via Festa del Perdono 7. I-20122 Milano, Italy. Correspondence to G.R. e-mail: giacomo.rizzolatti@unipr.it doi:10.1038/nrn2805 Published online 10 March 2010

\title{
The functional role of the parieto-frontal mirror circuit: interpretations and misinterpretations
}

\section{Giacomo Rizzolatti* and Corrado Sinigaglia}

Abstract | The parieto-frontal cortical circuit that is active during action observation is the circuit with mirror properties that has been most extensivelystudied. Yet, there remains controversy on its role in social cognition and its contribution to understanding the actions and intentions of other individuals. Recent studies in monkeys and humans have shed light on what the parieto-frontal cortical circuit encodes and its possible functional relevance for cognition. We conclude that, although there are several mechanisms through which one can understand the behaviour of other individuals, the parieto-frontal mechanism is the only one that allows an individual to understand the action of others 'from the inside' and gives the observer a first-person grasp of the motor goals and intentions of other individuals.

One of the most intriguing and exciting developments in neuroscience in recent years has been the discovery of a mechanism that unifies action perception and action execution $^{1-3}$. The essence of this mechanism - called the mirror mechanism - is the following: each time an individual observes another individual performing an action, a set of neurons that encode that action is activated in the observer's cortical motor system.

The mirror mechanism is present in many cortical areas and brain centres of birds, monkeys and humans. The basic functions of these areas and centres vary considerably, from song production to the organization of goal-directed motor acts, to emotional processes. Thus, like other basic mechanisms (for example, excitatory postsynaptic potentials), the functional role of the mirror mechanism depends on its anatomical location, with its function ranging from recognition of the song of conspecifics in birds ${ }^{4,5}$ to empathy in humans $\mathrm{s}^{6}$.

The aim of this article is not to review the vast literature on the mirror mechanism, but to focus on one specific circuit endowed with mirror properties: the parietofrontal action observation-action execution circuit. The reason for this choice is twofold. First, the proposed interpretation of the function of the parieto-frontal circuit as a mechanism that enables individuals to understand the actions and intentions of others (mirror-based action understanding) represented a paradigm shift in the classical view that these cognitive functions depend on higher-level mental processes. Second, mostly as a reaction to this new perspective, there have been attempts to interpret the functions of the action observation-action execution circuit in a way that minimizes or even denies its role in cognition. For these reasons, a review of the data on the mirror mechanism in the action observation-action execution network seems timely and necessary.

In this Review, we examine first what the parieto-frontal action observation-action execution circuit encodes in monkeys and humans and then discuss its possible functional relevance for cognition. After examining different views on these issues, we conclude that the parieto-frontal mechanism allows an individual to understand the actions of another individual 'from the inside' and gives the observing individual a first-person grasp of the motor goals and intentions of another individual.

\section{The parieto-frontal mirror network}

The monkey parieto-frontal network. The mirror mechanism was originally discovered in the ventral premotor cortex of the macaque monkey (area F5) ${ }^{1-3}$. Single-neuron recordings showed that this area contains neurons - mirror neurons - that discharge both when a monkey executes a specific motor act and when it observes another individual performing the same motor act. Mirror neurons do not fire in response to a simple presentation of objects, including food. Most of them do not respond or respond only weakly to the observation of the experimenter performing a motor act (for example, grasping) without a target object ${ }^{7}$.

Area F5 has recently been divided into three sectors: F5c, F5p and F5a ${ }^{8-9}$ (FIG. 1). Mirror neurons were 


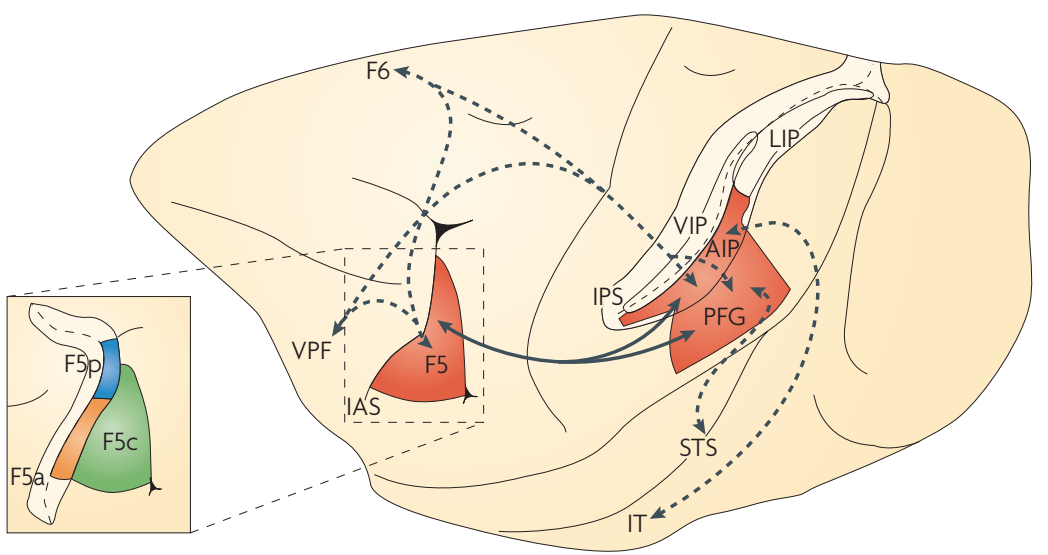

Figure 1 | The parieto-frontal mirror network. Lateral view of the macaque brain. The coloured areas represent the areas of the parieto-frontal circuit containing mirror neurons: the ventral premotor cortex (area F5), area PFG (located between parietal areas PF and PG) and the anterior intraparietal area (AIP). Note that the intraparietal sulcus (IPS) has been opened (light yellow) to show the areas inside. The parieto-frontal circuit receives high-order visual information from areas located inside the superior temporal sulcus (STS) and the inferior temporal lobe (IT). Neither of these temporal regions has motor properties. The parieto-frontal circuit is under control of the frontal lobe (area F6 or pre-supplementary motor area and the ventral prefrontal cortex (VPF)). The inset provides an enlarged view of area F5, showing also its sectors (F5a and F5p) buried inside the arcuate sulcus. IAS, inferior limb of the arcuate sulcus; LIP, lateral intraparietal area; VIP, ventral intraparietal area.

Mirror-based action understanding

The comprehension of an observed action based on the activation of a motor programme in the observer's brain. The observed action is understood 'from the inside' as a motor possibility, rather than 'from the outside' as a mere visual description.

Superior temporal sulcus (STS). This sulcus separates the superior temporal gyrus from the middle temporal gyrus. Some of the areas in the STS encode biological motion. Although connected with the parietal areas of the parieto-frontal mirror network, STS areas cannot be considered mirror areas because of their lack of motor properties.

Movement

A displacement of joints or body parts without a specific goal. It can be generated spontaneously or produced artificially by electrical or magnetic stimulation of motor areas. originally recorded in the cortical convexity that corresponds to $\mathrm{F} 5 \mathrm{c}^{1-3}$. However, functional MRI (fMRI) data showed that the other two areas also respond to observing a grasping action ${ }^{8}$.

Mirror neurons are also present in the rostral part of the inferior parietal lobule (IPL), particularly in area $\mathrm{PFG}^{10-12}$ and the anterior intraparietal area (AIP) $)^{9,13}$ (FIG. 1). Both these areas are heavily connected with F5: PFG mostly with F5c, and the AIP with F5a ${ }^{14}$. Both area PFG and the AIP receive higher-order visual information from the cortex located inside the superior temporal sulcus (STS) $)^{13-14}$. STS areas, like mirror areas, encode biological motion, but they lack motor properties. They are therefore not part of the mirror system in a strict sense. The AIP also receives connections from the middle temporal gyrus ${ }^{15}$. This input could provide the mirror areas with information concerning object identity. Finally, area F5 is connected with area F6 - the presupplementary motor area (pre-SMA) - and with the prefrontal cortex (area 46) ${ }^{16}$. The prefrontal cortex is also richly connected with the AIP ${ }^{16}$. The frontal inputs control the selection of self-generated and stimulus-driven actions according to the intentions of the agent ${ }^{17}$.

It was recently shown that, in addition to areas $\mathrm{PFG}$ and AIP, two other areas of the parietal lobe contain mirror neurons: the lateral intraparietal area and the ventral intraparietal area. The mirror properties of neurons in these areas are not the focus of this Review but are briefly discussed in BOX 1.

The human parieto-frontal network. There is convincing evidence that an action observation-action execution mirror circuit also exists in humans. This evidence comes from brain imaging, transcranial magnetic stimulation (TMS), electroencephalography (EEG) and magnetoencephalography (MEG) studies.

Brain imaging studies have shown that, as in the monkey, this action observation-action execution mirror circuit is formed by two main regions: the inferior section of the precentral gyrus plus the posterior part of the inferior frontal gyrus; and the inferior parietal lobule, including the cortex located inside the intraparietal sulcus ${ }^{18}$. Additional cortical areas (such as the dorsal premotor cortex and the superior parietal lobule) have also been occasionally found to be active during action observation and execution ${ }^{19-21}$. Although it is possible that their activation is due to a mirror mechanism, it is equally possible that it reflects motor preparation. In support of this interpretation are single-neuron data from monkeys showing that these areas are involved in covert motor preparation ${ }^{22-23}$. As for the superior parietal lobule, although its activation is typically absent in studies in which the experimenters use distal motor acts as visual stimuli, it is prominent when volunteers observe proximal arm movements that are directed to a particular location in space ${ }^{24}$.

Single-subject fMRI analyses have recently provided evidence that other cortical areas (for example, the primary and secondary somatosensory cortices and the middle temporal cortex) also become active during action observation and action execution ${ }^{21}$. It has been suggested ${ }^{21}$ that these activations outside of the 'classical' mirror areas are caused by additional mechanisms (for example, internal models) that are triggered by the mirror mechanism. These activations would enrich the information about the actions of other individuals that the mirror mechanism provides.

A tale of two populations. Some authors have recently argued that the activation of the same areas during action observation and action execution is not sufficient to prove the existence of the mirror mechanism in humans ${ }^{25}$. Instead, they have suggested that, in humans, motor areas have distinct, segregated populations of visual and motor neurons, the visual neurons discharging during action observation and the motor neurons during action execution. They proposed to use the 'repetitionsuppression' technique - that is, a technique based on the progressive reduction of a physiological response to repeated stimuli to prove this point ${ }^{25}$. If mirror neurons exist in humans, they should 'adapt' when the observation of a motor act is followed by the execution of that motor act, and vice versa.

The 'adaptation' effects are, in general, difficult to interpret ${ }^{26}$. Adaptation occurs at the synaptic level and should therefore be present only when information repeatedly reaches a neuron through the same or largely common pathways ${ }^{27}$. This input commonality is typically absent when mirror neurons are activated during action observation and execution. During action observation, the input to the parieto-frontal circuit arrives from higherorder visual areas (for example, the STS) ${ }^{16}$ whereas, during voluntary movement, it mostly comes from the frontal lobes ${ }^{17}$. The results of adaptation experiments therefore depend on the design of the experimental paradigm and 
on the stimuli used. These considerations could explain why the results of repetition-suppression experiments have been contradictory. Although some authors found evidence of the mirror mechanism in the parietal ${ }^{28}$ or the frontal nodes ${ }^{29}$, others obtained negative results ${ }^{30-31}$.

Regardless of the empirical data that may help to define some properties of the parieto-frontal mirror mechanism, the logic of the two-population story is flawed. Assuming that neurons in motor areas responding to action observation are merely visual neurons implies that motor areas contain a large number of 'displaced' visual neurons and that these neurons do not communicate with their 'neighbour' motor neurons. Both these assumptions are hard to reconcile with what is known about the organization of the cerebral cortex. Most importantly, TMS studies have shown a clear congruence between the observed motor act and the activated motor representation ${ }^{32-36}$. Thus, if higher-order sensory information describing a motor act reaches motor neurons that encode that same motor act, these motor neurons are mirror neurons by definition. Humans do not differ from monkeys in this respect.

What do parieto-frontal mirror neurons encode? Evidence for goal coding in monkeys. The crucial issue concerning the parieto-frontal mirror neurons is their role in cognition. If this mirror mechanism is fundamental to understanding actions and intentions, the classical view - that the motor system has a role only in movement generation - has to be rejected and replaced by the view that the motor system is also one of the major players in cognitive functions. To address this fundamental issue, a preliminary problem must first be solved: what do the parieto-frontal mirror neurons encode when they discharge in response to the observation of the actions of others?

A way to solve this problem is to examine what mirror neurons encode when they discharge during motor behaviour. What is recorded in single-neuron studies during both action execution and observation are action potentials - that is, neuronal output. Thus, having determined what neurons encode during the execution of an agent's own motor act, one also knows what they encode when they are triggered by the agent's observation of a motor behaviour of others.

Early experiments on area F5 found that most of the motor neurons in this area encode motor acts (that is, goal-related movements, such as grasping) rather than movements (that is, body-part displacements without a specific goal, such as finger flexion $)^{37-38}$. A recent study provided compelling evidence that this is the case ${ }^{39}$. This study describes single-neuron recordings from monkeys that were trained to grasp objects using two types of pliers: normal pliers, which require typical grasping movements of the hand, and 'reverse' pliers, which require hand movements executed in the reverse order (that is, first closing and then opening the fingers). The results showed that F5 neurons discharged during the same phase of grasping in both conditions, regardless of whether this involved opening or closing of the hand (FIG. 2).

The functional properties of IPL motor neurons are similar to those of F5 neurons: the goal of the executed motor acts is the parameter that is encoded by IPL neurons that fire during the execution of motor acts ${ }^{11,40-42}$.

The mirror neurons in F5 and IPL do not differ in their motor properties from parieto-frontal motor neurons that do not have visual properties ${ }^{1-3}$. Thus, when they fire in response to motor act observation, they send

\section{Box $1 \mid$ Mirror neurons in other parietal areas}

The cortical motor system in primates consists of a series of parieto-frontal circuits encoding different types of motor behaviour, among which are hand grasping, mouth and head movements, arm reaching and various types of eye movements. These circuits are activated by specific sets of sensory inputs that are conveyed to the parietal lobe from somatosensory, visual and auditory areas. In this Review, we focus on the mirror properties of the circuit formed in the monkey by parietal areas PFG and the anterior intraparietal area (AIP), and frontal area F5. Recently it was shown that two other areas of the parietal lobe also have mirror neurons: the lateral intraparietal area (LIP), which with the frontal eye field forms a circuit involved in the organization of eye movements; and the ventral intraparietal area (VIP), which is buried in the intraparietal sulcus and with frontal area F4 forms a circuit transforming somatosensory and visual stimuli that are presented around the monkey into head, mouth and arm movements (FIG. 1).

The properties of mirror neurons located in area LIP have been described by Shepherd and colleagues ${ }^{97}$. They found that a set of LIP neurons fired both when the monkey looked in the neuron-preferred direction and when it saw another monkey looking in the same direction. Interestingly, another subset of LIP neurons that discharged when the recorded monkey looked towards a certain direction was, by contrast, suppressed when the observed monkey looked in the same direction. The authors suggested that LIP mirror neurons contribute to the sharing of attention between individuals.

Mirror neurons in area VIP in the monkey have been described by Ishida and colleagues ${ }^{98}$. Previous studies showed that VIP neurons encode tactile and visual stimuli delivered in the peripersonal space of the monkey ${ }^{99,100}$. Ishida and colleagues showed that some of these neurons also respond to stimuli presented in the peripersonal space of an individual located approximately $1 \mathrm{~m}$ from the monkey and facing it. Although motor responses were not studied by this group, area VIP is connected with area F4, which represents peripersonal space and the neurons of which discharge during reaching movements. It is plausible, therefore, that neuronal responses that seem to be induced by visual stimuli actually represent potential motor acts directed towards specific body parts ${ }^{101-104}$. The study on VIP neurons is of great interest because it shows that the mirror mechanism of this area encodes body-directed rather than object-directed motor acts, thus opening fascinating possibilities for individuals to encode the body of others.

Thus, the function of mirror neurons is related to the motor properties of the areas in which they are located. This is also true for adjacent areas located in the same cortical region. 
$\sqrt{1}$
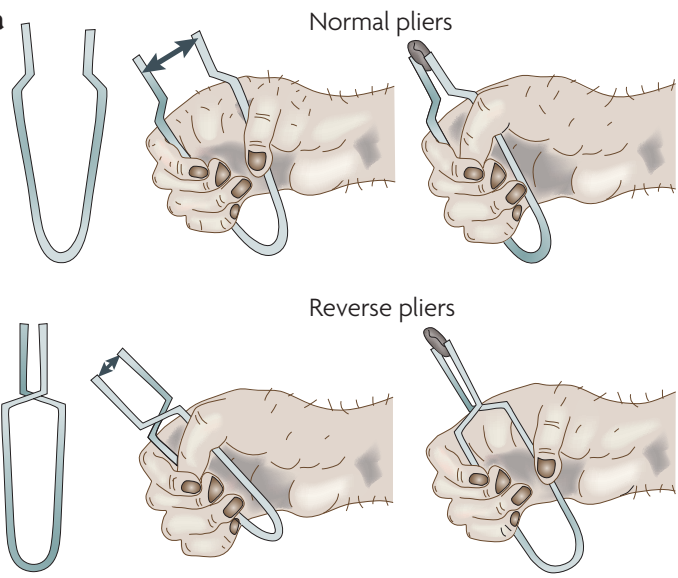

Reverse pliers

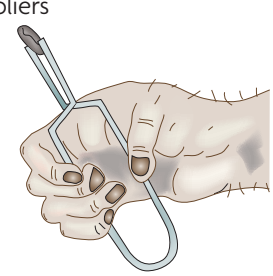

b Normal pliers
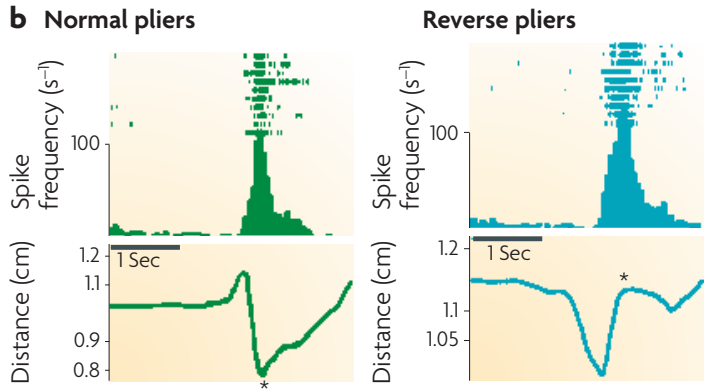

Figure 2 |Goal coding in the monkey premotor cortex. a|Schematic illustration of the experimental paradigm. To grasp the object with normal pliers, the monkey has to close its hand (top) whereas, with the reverse pliers, the monkey has to open its hand (bottom). The arrows indicate the direction of the motion of the tip of the pliers. b | Activity of a neuron recorded from area F5. Rasters and histograms illustrate the neuronal discharge during grasping with normal pliers (left) and reverse pliers (right). Both rasters and histograms are aligned with the end of the tip closure phase (asterisks). The traces below each histogram indicate the instantaneous hand position as recorded with a potentiometer and expressed as a function of the distance between the plier handles. Downwards traces indicate that the hand closes, whereas upwards traces indicate that the hand opens. Figure is reproduced, with permission, from REF. 39 @ (2008) National Academy of Sciences.

information about the goal of the observed motor acts. This information can be encoded with different degrees of generality: some mirror neurons (strictly congruent mirror neurons) fire when the observed and executed motor acts are the same (for example, grasping with precision grip), whereas other mirror neurons (broadly congruent mirror neurons) fire when the observed motor act has the same goal as the executed motor act (for example, grasping), but can be achieved in a different way (for example, with both precision and whole-hand grips $)^{43-44}$.

Recently, a single-neuron study investigated the effect of the spatial relationships between an agent and an observer, comparing F5 mirror neuron responses to motor acts performed near the monkey (in the peripersonal space) or outside its reach (in the extrapersonal space $)^{45}$ (FIG. 3). The results showed that many F5 mirror neurons were differentially modulated by the location of the observed motor act. Some neurons were selective for actions executed in the monkey's peripersonal space, whereas others were selective for stimuli in the extrapersonal space. These findings indicate that mirror neurons may encode the goal of the motor acts of another individual in an observer-centred spatial framework, thus providing the observer with crucial information for organizing their own future behaviour in cooperation or competition with the observed individuals.

Goal and single-movement coding in humans. In accordance with early findings ${ }^{46-49}$, a series of new fMRI studies provided strong evidence that the human parietofrontal mirror circuit encodes the goal of observed motor acts. Volunteers were instructed to observe video clips in which either a human or a robot arm grasped objects ${ }^{50}$. Despite differences in shape and kinematics between the human and robot arms, the parieto-frontal mirror circuit was activated in both conditions. Another group extended these results by investigating cortical activation in response to the observation of motor acts performed by a human hand, a robot hand or a tool ${ }^{51}$. Here, bilateral activation of a mirror network formed by intraparietal and ventral premotor cortex occured, regardless of the effector. In addition, the observation of tool actions produced a specific activation of a rostral sector of the left anterior supramarginal gyrus, suggesting that this sector specifically evolved for tool use.

Further evidence of goal encoding by the parietofrontal mirror circuit was obtained in an fMRI experiment in which two aplasic individuals, born without arms and hands, and control volunteers were asked to watch video clips showing hand actions ${ }^{52}$. All participants also performed actions with their feet, mouth and, in the case of controls, hands. The results showed that the parieto-frontal mirror circuit of aplasic individuals that was active during movements of the feet and mouth was also recruited by the observation of hand motor acts that they have never executed but the motor goals of which they could achieve using their feet or mouth.

The issue of whether the human parieto-frontal mirror network encodes motor goals was also addressed by fMRI and TMS studies investigating the activation of motor areas in subjects listening to action-related sounds. Hearing and categorizing animal vocalizations preferentially activated the middle portion of the superior temporal gyri bilaterally (a region that is not related to motor act coding), whereas hearing and categorizing sounds of tools that were manipulated by hands activated the parieto-frontal mirror circuit ${ }^{53}$. Similarly, it was shown that listening to the sound of hand and mouth motor acts activated the parieto-frontal mirror network ${ }^{54}$. This activation was somatotopically organized in the left premotor cortex and was congruent with the motor somatotopy of hand and mouth actions.

Unlike in monkeys, the parieto-frontal mirror circuit of humans also becomes active during the observation of individual movements $\mathrm{s}^{55-56}$. The initial evidence for this mechanism was based on TMS experiments which indicated that the observation of the movements of others results in an activation of the muscles involved in the 
execution of those movements ${ }^{32-36}$. Additional support comes from EEG and MEG studies showing that the observation of movements without a goal desynchronizes the rhythms recorded from motor areas ${ }^{57-64}$.

Recently, it was shown that mirror coding might depend on the content of the observed behaviour. Motor evoked potentials (MEPs) in response to TMS were recorded from the right opponens pollicis (OP) muscle in participants observing an experimenter either opening and closing normal and reverse pliers or using them to grasp objects ${ }^{65}$. The observation of tool movements (that is, opening and closing the pliers without grasping anything) activated a cortical representation of the hand movements involved in the observed motor behaviour. By contrast, the observation of the tool grasping action activated a cortical representation of the observed motor goal, irrespective of the individual movements and the order of movements required to achieve it. Together, these findings show that the human parieto-frontal mirror network encodes both motor acts and movements.

\section{Understanding the actions of others}

Cognitive functions of the parieto-frontal network: evidence and criticisms. Why should the motor system encode the goal of actions performed by others? From the discovery of mirror neurons, the interpretation of this finding was that they allow the observer to understand directly the goal of the actions of others ${ }^{1-3}$ : observing actions performed by another individual elicits a motor activation in the brain of the observer similar to that which occurs when the observer plans their own actions, and the similarity between these two activations allows the observer to understand the actions of others without needing inferential processing ${ }^{43-44}$.

In support of this view, two studies showed that the meaning of the motor acts of other individuals could be understood in the absence of visual information describing them. In one study, monkeys heard the sounds of a motor act (such as ripping a piece of paper) without seeing $i^{66}$; in the other study, the monkeys knew that behind a screen was an object and saw the experimenter's hand disappear behind the screen, but they could not see any hand-object interaction ${ }^{67}$. The results showed that in both experiments F5 mirror neurons in the monkeys fired in the absence of visual information describing the motor act of the experimenter. The neuronal activation therefore underpinned the comprehension of the goal of the motor act of the other individual, regardless of the sensory information that described that motor act.

This interpretation of the function of the parieto-frontal mirror mechanism has been challenged with objections and alternative proposals ${ }^{68-71}$. A key criticism has been advanced by Csibra ${ }^{69}$. He argued that the interpretation of a
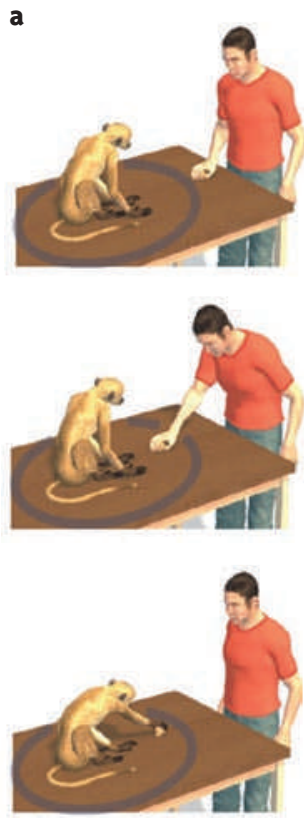

b
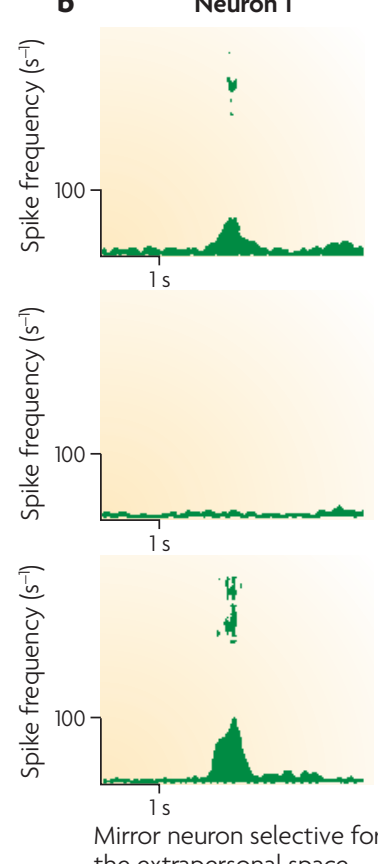

Neuron 2

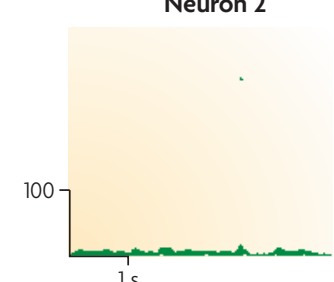

$1 \mathrm{~s}$

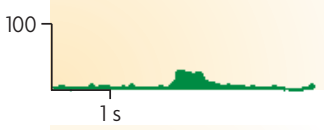

1

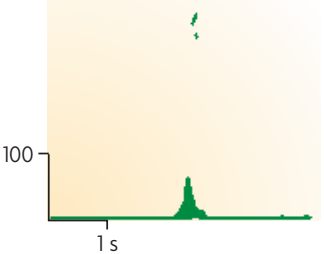

Mirror neuron selective for the peripersonal space

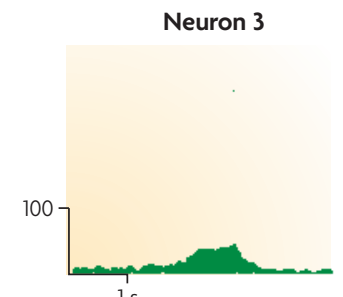

$1 \mathrm{~s}$
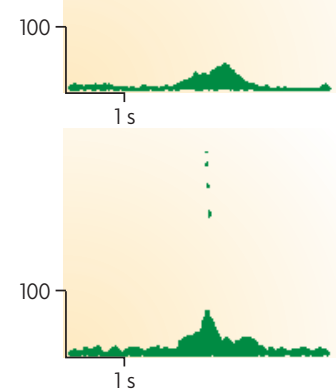

Classical mirror neuron

Figure 3 Mirror neuron responses in the monkey during observation of actions executed in the peripersonal and extrapersonal space. a Schematic illustration of the experimental paradigm. The monkey observes an experimenter grasping an object in its extrapersonal space (upper panel) and in its peripersonal space (middle panel). The monkey grasps an object located in front of it (lower panel). $\mathbf{b} \mid$ Responses of three neurons in the three experimental conditions. Neuron 1 responds selectively to the observation of action in the extrapersonal space, whereas neuron 2 responds to the observation of action in the peripersonal space. Neuron 3 does not exhibit space selectivity. All three neurons also discharge during action execution. Figure is reproduced, with permission, from REF. 45 ( ) (2009) American Association for the Advancement of Science. 
mirror neuron function in terms of action understanding contains a "tension" between "the claim that the mirror mechanism reflects nothing else but faithful duplication of the observed action" and "the claim that mirroring represents high-level interpretation of the observed action". In other words, if mirror activity represents a copy of the observed motor act, it is not sufficiently general to capture the goal of that motor act; conversely, if it is sufficiently general for goal understanding, it cannot be interpreted in terms of a direct matching mechanism between sensory and motor representations (see also REFS 70,71).

In the earlier studies on the mirror mechanism, it was indeed not clearly specified that the parieto-frontal mirror mechanism in humans is involved in two kinds of sensory-motor transformation - one mapping the observed movements onto the observer's own motor representation of those movements (movement mirroring), the other mapping the goal of the observed motor act onto the observer's own motor representation of that motor act (goal mirroring), as described above. By matching individual movements, mirror processing provides a representation of body part movements that might serve various functions (for example, imitation), but is devoid of any specific cognitive importance per se. By contrast, through matching the goal of the observed motor act with a motor act that has the same goal, the observer is able to understand what the agent is doing. This is true not only for the mirror neurons that are broadly congruent but also for those that are strictly congruent, because these neurons also do not encode the elementary aspects of a movement (for example, its kinematics), but respond to the goal of the observed motor acts ${ }^{44,56}$.

Typically, authors who play down or even deny the importance of the motor system for cognitive functions suggest that goal understanding is primarily due to cortical activation in the STS. This region, as described in a series of fundamental studies in monkeys ${ }^{72,73}$, is involved in the visual analysis of the actions of others. Several fMRI studies showed a similar role for the STS in humans (see REFS 74,75 for a review).

There is little doubt that STS neurons have an important role in encoding the behaviour of others. However, it is unlikely that the STS by itself mediates the processing of action understanding, relegating the parieto-frontal mirror network to an ancillary role in this function ${ }^{65}$ : among the neurons in various areas that become active during action observation, only those that can encode the goal of the motor behaviour of another individual with the greatest degree of generality can be considered to be crucial for action understanding, and the available evidence shows that this capacity for generalization characterizes the parietofrontal mirror neurons rather than STS cells. Indeed, parieto-frontal mirror neurons encode the goal of observed motor acts regardless of whether they are performed with the mouth, the hand or even with tools. Although STS neurons may encode some types of motor act, goal generalization such as is achieved by the parieto-frontal mirror neurons seems to be absent in the STS ${ }^{72,73}$.

Most importantly, there are theoretical reasons why STS neurons are unlikely to encode actions with the same degree of generality as parieto-frontal mirror neurons. If an STS neuron selectively encodes the visual features of a given hand action (for example, grasping), it is unclear how this neuron would also be able to encode selectively the visual features of a mouth performing the same motor act. One could postulate an association process similar to that described for the temporal lobe ${ }^{76,77}$. However, in the STS, the association would be between spatio-temporally adjacent visual representations of body part movements and not between visual representations of the same motor goal achieved by different effectors. By contrast, parieto-frontal mirror neurons - owing to their motor nature and the fact that they encode the goal of motor acts - can be triggered by different visual stimuli (for example, hand and mouth actions) that have a common goal (for example, grasping). Only the presence of a 'motor scaffold' that provides the goal-related aspects of observed actions can allow this generalization; such generalization cannot be achieved by mere visual association.

A recent study provides empirical evidence in favour of this point ${ }^{78}$. The study was based on a TMS adaptation paradigm ${ }^{79}$. Participants were presented with 'adaptation-inducing' movies of a hand or foot acting on various objects and asked to respond as quickly as possible to a picture of a motor act similar to that of the movie. TMS pulses were delivered over the ventral premotor cortex bilaterally, over the left IPL and over the left STS. The results showed that the delivery of TMS over both premotor and IPL cortices shortened the reaction times to 'adapted' motor acts regardless of which effector performed the observed motor act; by contrast, TMS stimulation of the STS shortened the reaction times to 'adapted' motor acts only if the same effector executed the act in the movie and in the test picture.

Understanding actions from the inside. Another argument against the role of mirror neurons in action understanding is that there are several behavioural instances in which individuals understand the actions of others even if they are unable to perform them. For example, macaques can react to the observation of humans making the gesture of throwing objects overhand towards them ${ }^{80}$. It was proposed that, although monkeys never throw objects overhand, they could nevertheless understand the action they saw because they analysed the various visual elements of the observed actions and applied some form of inferential reasoning.

However, this argument would only be valid if the parieto-frontal mirror mechanism consisted solely of strictly congruent mirror neurons. As the authors of the study themselves recognize ${ }^{80}$, the capacity of broadly congruent mirror neurons to generalize the goal of motor acts might account for the observed phenomenon. Given that broadly congruent mirror neurons may generalize from a hand action to actions performed with tools, even when they are as bizarre as reverse pliers, it is plausible that they could equally generalize from one type of throwing to another.

There is no doubt that, in some cases, understanding the motor behaviour of others might require a mechanism different from mirroring. A typical example is the capacity 
of humans to recognize the actions of animals that do not belong to the human motor repertoire and cannot be captured by a motor generalization. Evidence for a nonmirror mechanism in action recognition was provided by an fMRI study in which volunteers were presented with video clips showing motor acts that did or did not belong to the human motor repertoire ${ }^{81}$. Although all volunteers recognized the observed motor acts regardless of whether or not they belonged to their own motor repertoire, no activation of parieto-frontal mirror areas was found in response to acts that did not belong to their motor repertoire (for example, a dog barking). The areas that became active in such cases were occipital visual and STS areas. By contrast, the sight of motor acts that were within the motor repertoire of the observer (for example, a dog biting) recruited the parieto-frontal mirror network.

These data indicate that the recognition of the motor behaviour of others can rely on the mere processing of its visual aspects. This processing is similar to that performed by the 'ventral stream' areas for the recognition of inanimate objects. It allows the labelling of the observed behaviour, but does not provide the observer with cues that are necessary for a real understanding of the conveyed message (for example, the communicative intent of the barking dog). By contrast, when the observed action impinges on the motor system through the mirror mechanism, that action is not only visually labelled but also understood, because the motor

\section{Box 2 | Some (pseudo) problems for the mirror mechanism}

In addition to the main objections to the role of the parieto-frontal mechanism in action understanding discussed in the main text, other 'problems' have been offered as evidence against the notion that the parieto-frontal mirror mechanism is involved in action understanding ${ }^{105}$. One problem derives from a transcranial magnetic stimulation experiment ${ }^{106}$ in which individuals were trained to move their fingers in a manner incongruent to that of the 'teacher'. After training, motor-evoked potentials were greater in the muscle involved in the incongruent response than in the muscle involved in the congruent one. This incongruence, contrary to the authors' claim, does not necessarily demonstrate the discovery of a new class of mirror neurons. It probably shows that one can inhibit a natural (mirror) response and voluntarily organize a different response to comply with instructions. However, regardless of what this experiment tells us about the role of the mirror mechanism in imitation, its results are unrelated to action understanding. The reason is that, in the task, there was nothing to understand: the investigated movements were meaningless.

A second argument is even more surprising. The claim ${ }^{105}$ is that, if the human parieto-frontal mirror system were involved in action understanding, motor syndromes following its damage (for example, ideomotor apraxia) would be accompanied by a deficit in action recognition. The assumption is that there is a one-to-one correspondence between motor and mirror sectors in the parieto-frontal circuit. This assumption is wrong. As clearly shown by electrophysiological mapping, there are motor sectors in the monkey inferior parietal lobule (and even in area PFG) with and without mirror neurons. Thus, dissociations between motor deficits and action understanding deficits can and do occur ${ }^{107}$. It is the opposite result - that specific action recognition deficits can occur following motor circuit damage $\mathrm{e}^{108}$ — that is striking and that provides a compelling argument for the crucial role of mirror neurons in this function.

Finally, saxophone playing has been used as an example to show that the mirror view of action understanding is "untenable": no motor competence is required to understand that someone is playing a saxophone ${ }^{105}$. This is true, but such competence leads to a different understanding of saxophone playing. The non-motor-based understanding implies a mere semantic knowledge of what a saxophone is for, whereas the motor experience allows an individual to understand what saxophone playing really means - that is, it provides a musical knowledge 'from the inside'. representation of its goal is shared by the observer and the agent. In other words, the observed action is understood from the inside as a motor possibility and not just from the outside as a mere visual experience (BOX 2).

\section{Understanding motor intentions of others}

From motor goals to motor intentions. The properties of parieto-frontal mirror neurons described above indicate that their activity reflects what is going on in the "here and now' However, there is evidence that parietal and frontal mirror neurons are involved in encoding not only the observed motor acts but also the entire action of which the observed motor act is part. Monkeys were trained to grasp objects with two different motor intentions: to place them into a container or to bring them to their mouth ${ }^{11}$. After training, motor neurons in the IPL that encode grasping were studied in the two set-ups. The results showed that the majority of these neurons discharged with an intensity that varied according to the action in which the motor act was embedded ('actionconstrained motor neurons'). This finding implies that the IPL contains 'chains' of neurons in which each neuron encodes a given motor act and is linked to others that are selective for another specific motor act. Together, they encode a specific action (for example, grasping for eating).

A striking result of this study was that many of these action-constrained motor neurons have mirror properties. When tested in the two set-ups described above, the majority of these neurons were differently activated depending on the action to which the observed motor act belonged ('action-constrained mirror neurons'). This finding indicates that, in addition to describing what the observed individual is doing (for example, grasping), IPL mirror neurons also help the observer to explain why the individual is performing the action, owing to chained organization in the IPL. That is, IPL mirror neurons enable the observer to recognize the agent's motor intention.

A recent study demonstrated that action-constrained neurons are also present in area F5 (REF. 82). The comparison of F5 and IPL (specifically area PFG) mirror neuron properties revealed no clear differences in their capacity to encode the motor intentions of others.

Evidence that the parieto-frontal mirror circuit in humans is also involved in intention encoding was first provided by an fMRI experiment consisting of three conditions ${ }^{83}$. In the first (the 'context condition') the volunteers saw a photo of some objects arranged as for an ongoing breakfast or arranged as though the breakfast had just finished; in the second (the 'action condition'), the volunteers saw a photo of a hand grasping a mug without any context; in the third (the 'intention condition') they saw photos showing the same hand actions within the two contexts. In this condition, the context provided clues for understanding the intention of the motor act. The results showed that the intention condition induced a stronger activation than the other two conditions in the caudal inferior frontal gyrus of the right hemisphere.

An activation of the right parieto-frontal mirror circuit during intention understanding was also described in a repetition-suppression fMRI experiment ${ }^{84}$. 
Participants were presented with movies showing motor actions (for example, pushing or pulling a lid) that could lead to the same or to different outcomes (for example, opening or closing a box). The results showed that the responses in the right IPL and right inferior frontal cortex were 'suppressed' when participants saw movies of motor actions that had the same outcome, regardless of the individual movements involved. Responses in these regions were not influenced by the kinematics parameters of the observed motor action.

Brain imaging experiments allow the cortical substrate of a given function to be located, but they do not give information about the mechanism underlying the function. Cattaneo and colleagues tested whether the understanding of motor intention in humans might be based on the 'chain mechanism' described in the monkey ${ }^{85}$. Participants were asked to grasp a piece of food and eat it or to grasp a piece of food and place it in a container. In another condition, they had to observe an experimenter performing the same actions. In both the execution and the observation condition, the electromyographic activity of the mylohyoid muscle - a muscle involved in mouth opening - was recorded. Both the execution and the observation of the eating action produced a marked increase of mylohyoid muscle activity as early as the 'reaching' phase, whereas no mylohyoid muscle activity was recorded during the execution and the observation of the placing action. This indicates that, as soon as the action starts, the entire motor programme for a given action is activated. Interestingly, the observers also seem to have a motor copy of this programme. This 'intrusion' allows them to predict what action the agent is going to execute from the first observed motor act and thus to understand the agent's motor intention.

Finally, there is evidence that the mirror mechanism, possibly located in this case in the fronto-mesial areas, also has a role in setting up an anticipatory representation of the motor behaviour of another individual. It has been shown that the 'Bereitschaftspotential', an electrophysiological marker of the readiness to act ${ }^{86}$, occurs not only when an individual actively performs a motor act, but also when the nature and the onset time of an upcoming action performed by another individual is predictable on the basis of a visual cue ${ }^{87}$.

Mirroring intentions and inferring reasons. The studies reviewed above indicate that the parieto-frontal mirror network may subserve the understanding of the motor intention underlying the actions of others. This capacity represents a functional property of the parieto-frontal mirror network that further distinguishes it from those of visual areas. Indeed, it is difficult to imagine how motor intention understanding could be based on visual processing alone, including visual processing that is carried out in higher-order visual areas such as the STS. It is true that some STS neurons are selective for a sequence of stimuli. For example, in contrast to classical visual neurons that respond to a specific static stimulus, some STS neurons respond to the static view of a body only when this stimulus occurs after a certain movement (for example, walk and stop $)^{88}$. However, despite this fascinating property, these neurons do not give information about the agent's motor intention: they describe a given motor act according to a previous motor behaviour, but they do not provide information about the motor intention underlying that motor act.

This does not mean that the parieto-frontal mirror mechanism mediates all varieties of intention understanding. Intention understanding is a multi-layer process involving different levels of action representation, from the motor intention that drives a given chain of motor acts to the propositional attitudes (beliefs, desires and so on) that - at least in humans - can be assumed to explain the observed behaviour in terms of its plausible psychological reasons.

We provide an example to clarify this point. Mary is interacting with an object (for example, a cup). According to how she is grasping the cup, we can understand why she is doing it (for example, to drink from it or to move it). This kind of understanding can be mediated by the parieto-frontal mirror mechanism by virtue of its motor chain organization. However, the mirror mechanism is not able to provide us with the reasons that might underlie the motor intention of Mary (for example, she grasped the cup to drink from it because she was thirsty or because she wanted some caffeine, or she did it to please her friends). Understanding the reasons behind an agent's motor intention requires additional inferential processes ${ }^{89-91}$.

Recent empirical data confirmed these considerations. They showed that, although the parieto-frontal mirror mechanism is active in all conditions in which the motor task has to be directly understood, when volunteers were required to judge the reasons behind the observed actions, there was an activation of a sector of the anterior cingulate cortex and of other areas of the so-called 'mentalizing network' ${ }^{\text {'2 }}$. Activation of the same network was also shown in a study that investigated unusual actions performed in implausible versus plausible contexts $^{93}$, as well as in a study on the neural basis of reason inference in non-stereotypical actions ${ }^{94}$.

As there are different levels of action representation, there should be diverse neural mechanisms subserving these different levels of intention understanding. Understanding motor intention relies on the parietofrontal mirror mechanism and the motor chain organization of the cortical motor system. Understanding the reason behind motor intention seems to be localized in cortical areas - the temporal parietal junction and a part of the anterior cingulate gyrus - that have not as yet been shown to have mirror properties. There have been theoretical attempts to integrate these two ways of understanding the intentions of others ${ }^{95-96}$. Nonetheless, unlike for the mirror mechanism, there are currently no neurophysiological data that can explain how the 'mentalizing network' might work.

\section{Conclusions}

The mirror mechanism is a neurophysiological finding that has raised considerable interest over the past few years. It provides a basic mechanism that unifies action production and action observation, allowing the understanding of the actions of others from the inside. 


\section{Box 3 | The mirror mechanism and autism}

a
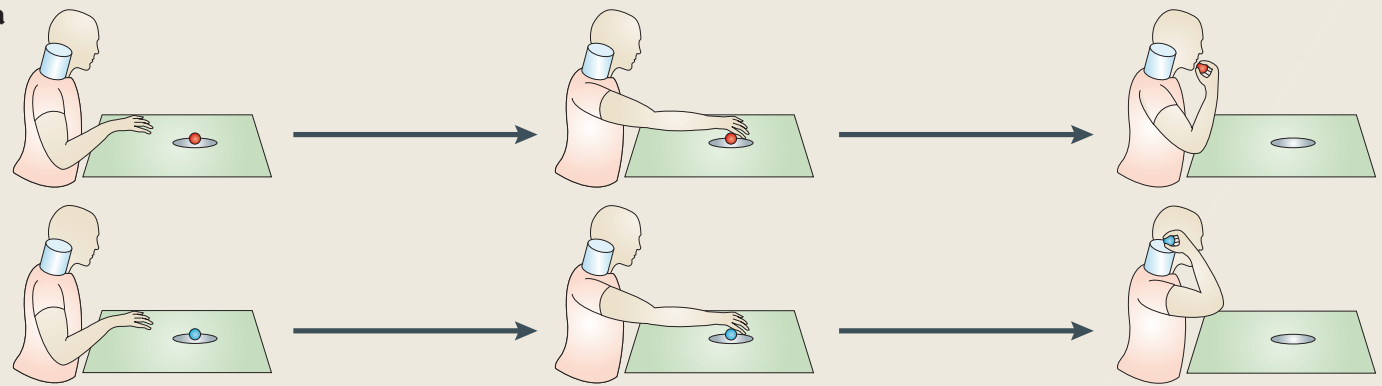

b Typically developing children
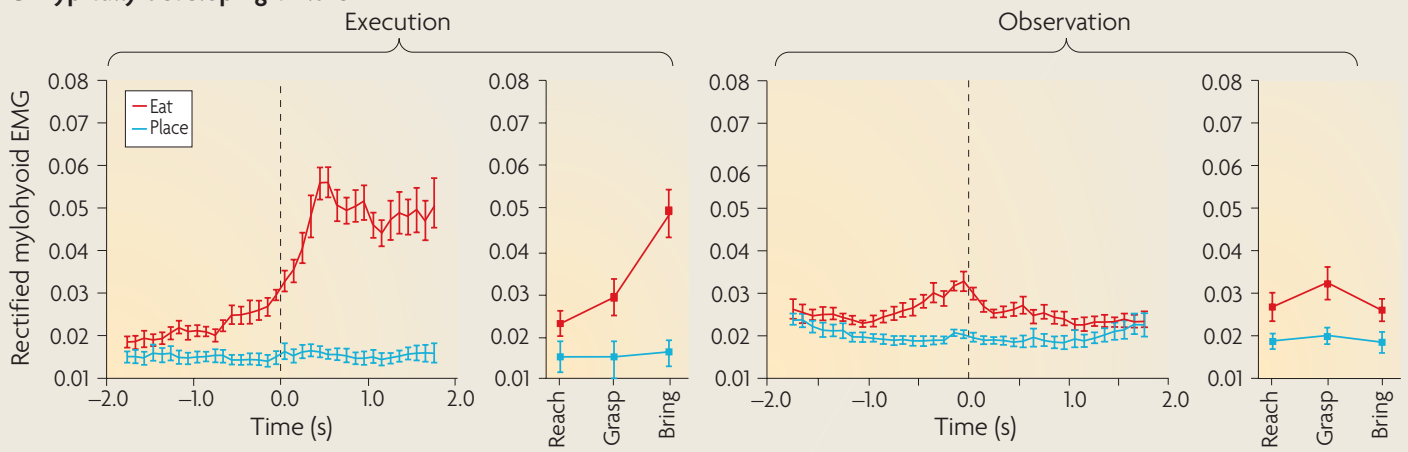

c Children with ASD
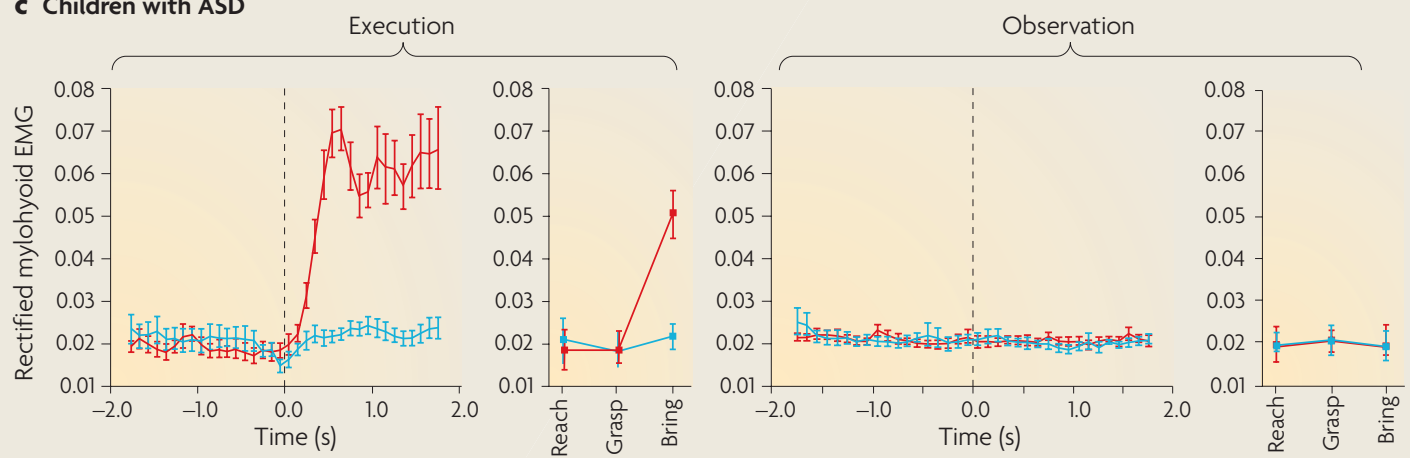

Autistic spectrum disorder (ASD) is a syndrome characterized by impairment in social skills, communicative abilities, emotional responses and motor behaviour ${ }^{109-113}$. Over the past few years, a number of electrophysiological and brain imaging experiments ${ }^{62,114-117}$ showed that individuals with ASD have an impairment of the mirror mechanism, suggesting that the social 'aloneness' that is typical of an individual with ASD might result from this deficit ${ }^{18-120}$. Recently, some behavioural studies have challenged this view ${ }^{121-123}$. The studies showed that the capacity of children with ASD to understand the goal of observed motor acts - a function of mirror neurons - is preserved and that the mirror hypothesis of ASD should therefore be considered incorrect ${ }^{124}$.

How can the discrepancy between imaging and electrophysiological findings and behavioural data be explained? An answer has been provided by a study in which children with ASD were asked to grasp a piece of food either for eating or for placing in a container (see the figure, part a) and, in another set-up, to observe an experimenter performing these actions ${ }^{85}$. The activity of the mylohyoid $(\mathrm{MH})$ muscle, which is involved in mouth opening, was recorded to produce an electromyograph (EMG). Unlike typically developing children, in whom $\mathrm{MH}$ activation was already present during the reaching and grasping phases of the grasping-for-eating action (see the figure, part $\mathbf{b}$, left), children with ASD showed MH activation only during the bringing-to-the-mouth phase (see the figure, part c, left). Furthermore, typically developing children exhibited MH activation when observing a grasping-for-eating action, whereas children with ASD did not (see the figure, parts $\mathbf{b}$ and $\mathbf{c}$, right). The interpretation of these data is straightforward: children with ASD have a severe impairment in motor organization that includes a deficit in chaining motor acts into intentional actions. Thus, during action observation the intentional motor chains are not activated. Therefore, the intentions of others do not 'intrude' into the mirror system of children with ASD and these intentions are not understood 'from the inside' but only 'from the outside'. This interpretation is supported by a recent study showing that to understand the intentions of other people, children with ASD do not rely on the observed motor behaviour but on the semantics of the object that is being manipulated or on the context in which the motor act takes place ${ }^{125}$. Figure is reproduced, with permission from REF. 85 (c) (2007) National Academy of Sciences. 
Such motor-based understanding seems to be a primary way in which individuals relate to one another, as shown by its presence not only in humans and monkeys, but also in evolutionarily distant species, such as swamp sparrows ${ }^{4}$ and zebra finches ${ }^{5}$. Furthermore, this mechanism indicates the existence of a profound natural link between individuals that is crucial for establishing inter-individual interactions. Finally, preliminary evidence suggests that the impairment of this natural link may be one of the causes of the striking inability of people with autism to relate to other individuals (BOX 3).
1. di Pellegrino, G., Fadiga, L., Fogassi, L., Gallese, V. \& Rizzolatti, G. Understanding motor events: a neurophysiological study. Exp. Brain Res. 91 176-180 (1992)

2. Gallese, V., Fadiga, L., Fogassi, L. \& Rizzolatti, G. Action recognition in the premotor cortex. Brain 119, 593-609 (1996).

3. Rizzolatti, G., Fadiga, L., Gallese, V. \& Fogassi, L. Premotor cortex and the recognition of motor actions. Cogn. Brain Res. 3, 131-141 (1996). Prather, J. F., Peters, S., Nowicki, S. \& Mooney, R. Precise auditory-vocal mirroring in neurons for learned vocal communication. Nature 451, 249-250 (2008). This accurate and elegant study was the first to show the existence of the mirror mechanism in non-primates.

5. Keller, G. B. \& Hahnloser, R. H. Neural processing of auditory feedback during vocal practice in a songbird. Nature 457, 187-190 (2009).

6. Gallese, V., Keysers, C. \& Rizzolatti, G. A unifying view of the basis of social cognition. Trends Cogn. Sci. 8, 396-403 (2004).

7. Kraskov, A., Dancause, N., Quallo, M. M., Shepherd, S. $\&$ Lemon, R. N. Corticospinal neurons in macaque ventral premotor cortex with mirror properties: a potential mechanism for action suppression? Neuron 64, 922-930 (2009).

8. Nelissen, K., Luppino, G., Vanduffel, W., Rizzolatti, G. ¿ Orban, G. A. Observing others: multiple action representation in the frontal lobe. Science 310 332-336 (2005)

9. Belmalih, A., Borra, E., Gerbella, M., Rozzi, S. \& Luppino, G. Connections of architectonically distinct subdivisions of the ventral premotor area F5 of the macaque. $37^{\text {th }}$ Annual Meeting of the Society for Neuroscience, San Diego, California. Abstract 636.5 (2007).

10. Gallese, V., Fadiga, L., Fogassi, L. \& Rizzolatti, G. in Common Mechanisms in Perception and Action: Attention and Performance Vol. 19 (eds Prinz, W. \& Hommel, B.) 334-355 (Oxford University Press, New York, 2002).

11. Fogassi, L. et al. Parietal lobe: from action organization to intention understanding. Science 302, 662-667 (2005).

12. Rozzi, S., Ferrari, P. F., Bonini, L., Rizzolatti, G. \& Fogassi, L. Functional organization of inferior parietal lobule convexity in the macaque monkey: electrophysiological characterization of motor, sensory and mirror responses and their correlation with cytoarchitectonic areas. Eur. J. Neurosci. 28, 1569-1588 (2008)

13. Rizzolatti, G., Fogassi, L. \& Gallese, V. in The Cognitive Neuroscience (ed. Gazzaniga, M.), 625-640 (MIT Press, Cambridge, Massachusetts, 2009).

14. Rozzi, S. et al. Cortical connections of the inferior parietal cortical convexity of the macaque monkey. Cereb. Cortex 16, 1389-1417 (2006)

15. Borra, E. et al. Cortical connections of the macaque anterior intraparietal (AIP) area. Cereb. Cortex 18, 1094-1111 (2008)

16. Rizzolatti, G. \& Luppino, G. The cortical motor system Neuron 31, 889-901 (2001).

17. Fuster, J. M. The Prefrontal Cortex 4th edn (Academic Press, London, 2008)

18. Rizzolatti, G. \& Craighero, L. The mirror neuron system. Annu. Rev. Neurosci. 27, 169-192 (2004).

19. Grézes, J., Armony, J. L., Rowe, J. \& Passingham, R. E. Activations related to "mirror" and "canonical" neurones in the human brain: an fMRI study. Neuroimage 18, 928-937 (2003)

20. Buccino, G. et al. Neural circuits underlying imitation learning of hand actions: an event-related fMRI study. Neuron 42, 323-334 (2004).

21. Gazzola, V. \& Keysers, C. The observation and execution of actions share motor and somatosensory voxels in all tested subjects: single subject analyses of unsmoothed fMRI data. Cereb. Cortex 19, 1239-1255 (2009).
22. Kalaska, J. F. \& Crammond, D. J. Deciding not to go: neuronal correlates of response selection in a go/nogo task in primate premotor and parietal cortex Cereb. Cortex 5, 410-428 (1995).

23. Crammond, D. J. \& Kalaska, J. F. Prior information in motor and premotor cortex: activity during the delay period and effect on pre-movement activity. J. Neurophysiol 84, 986-1005 (2000).

24. Filimon, F., Nelson, J. D., Hagler, D. J. ¿ Sereno, M. L. Human cortical representations for reaching: mirror neurons for execution, observation, and imagery. Neuroimage 37, 1315-1328 (2007).

25. Dinstein, I., Thomas, C., Behrmann, M. \& Heger, D. I. A mirror up to nature. Curr. Biol. 18, R13-R18 (2008).

26. Bartels, A., Logothetis, N. K. \& Moutoussis, K fMRI and its interpretations: an illustration on directional selectivity in area V5/MT. Trends Neurosci. 31, 444-453 (2008)

27. Sawamura, H., Orban, G. A. \& Vogels, R. Selectivity of neural adaptation does not match response selectivity: a single-cell study of the fMRI adaptation paradigm. Neuron 49, 307-318 (2006)

28. Chong, T. T., Cunnington, R., Williams, M. A Kanwisher, N. \& Mattingley, J. B. fMRI adaptation reveals mirror neurons in human inferior parietal cortex. Curr. Biol. 18, 1576-1580 (2008)

29. Kilner, J. M., Neal, A., Weiskopf, N., Friston, K. J. \& Frith, C. Evidence of mirror neurons in human inferior frontal gyrus. J. Neurosci. 29, 10153-10159 (2009).

30. Dinstein, I., Hasson, U., Rubin, N. \& Heeger, D. J. Brain areas selective for both observed and executed movements. J. Neurophysiol. 98, 1415-1427 (2007).

31. Lignau, A., Gesierich, B. ¿ Caramazza, A. Asymmetric fMRI adaptation reveals no evidence for mirror neurons in humans. Proc. Natl Acad. Sci. USA 106, 9925-9930 (2009).

32. Fadiga, L., Fogassi, L., Pavesi, G. \& Rizzolatti, G. Motor facilitation during action observation: a magnetic stimulation study. J. Neurophysiol. 73, 2608-2611 (1995).

33. Strafella, A. P. \& Paus, T. Modulation of cortical excitability during action observation: a transcranial magnetic stimulation study. Neuroreport 11 2289-2292 (2000).

34. Gangitano, M., Mottaghy, F. M. \& Pascual-Leone, A. Phase-specific modulation of cortical motor output during movement observation. Neuroreport 12, 1489-1492 (2001)

35. Maeda, F., Kleiner-Fisman, G. \& Pascual-Leone, A Motor facilitation while observing hand actions: specificity of the effect and role of observer's orientation. J. Neurophysiol. 87, 1329-1335 (2002).

36. Borroni, P., Montagna, M., Cerri, G. \& Baldissera, F. Cyclic time course of motor excitability modulation during the observation of a cyclic hand movement. Brain Res. 1065, 115-124 (2005).

37. Rizzolatti, G et al. Functional organization of inferior area 6 in the macaque monkey: II. Area F5 and the control of distal movements. Exp. Brain Res. 71, 491-507 (1988)

38. Kakei, S., Hoffman, D. S. \& Strick, P. L. Direction of action is represented in the ventral premotor cortex. Nature Neurosci. 4, 1020-1025 (2001)

39. Umiltà, M. A. et al. How pliers become fingers in the monkey motor system. Proc. Natl Acad. Sci. USA 105, 2209-2213 (2008)

This single-neuron recording study provides crucial evidence that the ventral premotor cortex (area F5) encodes the goal of motor acts.

40. Hyvärinen, J. Posterior parietal lobe of the primate brain. Physiol. Rev. 62, 1060-1129 (1982).

41 Sakata, H., Taira, M., Murata, A \& Mine, S. Neural mechanisms of visual guidance of hand action in the parietal cortex of the monkey. Cereb. Cortex 5, 429-438 (1995)

42. Jeannerod, M., Arbib, M. A., Rizzolatti, G. \& Sakata, H. Grasping objects: the cortical mechanisms of visuomotor transformation. Trends Neurosci. 18, 314-320 (1995).
43. Rizzolatti, G., Fogassi, L. \& Gallese, V. Neurophysiological mechanisms underlying the understanding and imitation of action. Nature Rev. Neurosci. 2, 661-670 (2001).

44. Rizzolatti, G. \& Sinigaglia, C. Mirrors in the Brain. How our Minds Share Actions and Emotions (Oxford University Press, Oxford/New York, 2008). An exhaustive review and discussion of the cognitive functions of the motor system.

45. Caggiano, V., Fogassi, L., Rizzolatti, G., Thier, P. \& Casile, A. Mirror neurons differentially encode the peripersonal and extrapersonal space of monkeys. Science 324, 403-406 (2009).

This single-neuron recording study shows that mirror-based goal encoding can be modulated by the position in space in which the observed action takes place and by the possibility of interacting with the object that is acted upon.

46. Rizzolatti, G. et al. Localization of grasp representation in humans by PET: 1. Observation versus execution. Exp. Brain Res. 11, 246-252 (1996).

47. Grafton, S. T., Arbib, M. A., Fadiga, L. \& Rizzolatti, G. Localization of grasp representations in humans by positron emission tomography: 2. Observation compared with imagination. Exp. Brain Res. 12, 103-111 (1996).

48. Buccino, G. et al. Action observation activates premotor and parietal areas in a somatotopic manner: an fMRI study. Eur. J. Neurosci. 13, 400-404 (2001)

49. Decety, J., Chaminade, T., Grezes, J. \& Meltzoff, A. N. A PET exploration of the neural mechanism involved in reciprocal imitation. Neuroimage 15, 265-272 (2002).

50. Gazzola, V., Rizzolatti, G., Wicker, B. \& Keysers, C The anthropomorphic brain: the mirror neuron system responds to human and robotic actions. Neuroimage 35, 1674-1684 (2007)

51. Peeters, R. et al. The representation of tool use in humans and monkeys: common and unique human features. J. Neurosci. 29, 11523-11539 (2009). An elegant fMRI study showing that the observation of tool actions activates the parieto-frontal mirror circuit plus a specific parietal region involved in both tool use and the understanding of tool use.

52. Gazzola, V. et al. Aplasics born without hands mirror the goal of hand actions with their feet. Curr. Biol. 17, 1235-1240 (2007)

This fMRI study provides one of the best demonstrations that the parieto-frontal mirror network in humans encodes the goal of the observed motor acts.

53. Lewis, J. W., Brefczynski, J. A., Phinney, R. E., Janik, J. J. \& De Yoe, E. A. Distinct cortical pathways for processing tool versus animal sounds. J. Neurosci. 25, 5148-5158 (2005)

54. Gazzola, V., Aziz-Zadeh, L. \& Keysers, C. Empathy and the somatotopic auditory mirror system in humans. Curr. Biol. 16, 1824-1829 (2006)

55. Lui, F. et al. Neural substrates for observing and imaging non object-directed actions. Soc. Neurosci. 3, 261-275 (2008)

56. Rizzolatti, G., Fadiga, L., Fogassi, L. \& Gallese, V. Resonance behaviors and mirror neurons. Arch. Ital. Biol. 137, 85-100 (1999).

57. Altschuler, E. L., Vankov, A., Wang, V. Ramachandran, V. S. \& Pineda, J. A. Person see, person, do: human cortical electrophysiological correlates of monkey see monkey do cells. $27^{\text {th }}$ Annua Meeting of the Society for Neuroscience, New Orleans, Los Angeles. Abstract 719.17 (1997).

58. Hari, R. et al. Activation of human primary motor cortex during action observation: a neuromagnetic study. Proc. Natl Acad. Sci. USA 95, 15061-15065 (1998).

59. Cochin, S., Barthelemy, C., Lejeune, B., Roux, S. \& Martineau, J. Perception of motion and qEEG activity in human adults. Electroencephalogr. Clin. Neurophysiol. 107, 287-295 (1998). 
60. Pineda, J. A. The functional significance of mu rhythms: translating "seeing" and "hearing" into “doing”. Brain Res. Brain Res. Rev. 50, 57-68 (2005)

61. Oberman, L. M. et al. EEG evidence for mirror neuron dysfunction in autism spectrum disorders. Brain Res. Cogn. Brain Res. 24, 190-198 (2005).

62. Oberman, L. M., Pineda, J. A. \& Ramachandran, V. S. The human mirror neuron system: a link between action observation and social skills. Soc. Cog. Affect. Neurosci. 2, 62-66 (2007).

63. Kilner, J. M., Marchant, J. L. \& Frith, C. D. Relationship between activity in human primary motor cortex during action observation and the mirror neuron system. PLoS ONE 4, e4925 (2009).

64. Perry, A. \& Bentin, S. Mirror activity in the human brain while observing hand movements: a comparison between EEG desynchronization in the mu-range and previous fMRI results. Brain Res. 1282, 126-132 (2009).

65. Cattaneo, L., Caruana, F., Jezzini, H. \& Rizzolatti, C. Representation of goal and movements without overt motor behavior in the human motor cortex: a TMS study. J. Neurosci. 29, 11134-11138 (2009).

66. Kohler, E. et al. Hearing sounds, understanding actions: action representation in mirror neurons. Science 297, 846-848 (2002)

67. Umiltà, M. A. et al. "I know what you are doing": a neurophysiological study. Neuron 32, 91-101 (2001)

68. Wilson, M. \& Knoblich, G. The case of motor involvement in perceiving conspecifics. Psychol. Bull. 131, 460-473 (2005)

69. Csibra, G. in Sensorimotor Foundations of Higher Cognition. Attention and Performance XII (eds Haggard, P., Rosetti, Y. \& Kawato, M.) 453-459 (Oxford University Press, Oxford, 2007). The most interesting and critical assessment of the mirror mechanism and its function in cognition.

70. Jacob, P. What do mirror neurons contribute to human social cognition? Mind Lang. 23, 190-223 (2008).

71. Jacob, P. The tuning-fork model of human social cognition: a critique. Conscious. Cogn 18, 229-243 (2009).

72. Perrett, D. I. et al. Frameworks of analysis for the neural representation of animate objects and actions. J. Exp. Biol. 146, 87-113 (1989).

73. Jellema, T. \& Perrett, D. I. in The Cognitive Neuroscience of Social Behavior (eds Easton, A. \& Emery, N. J.) 81-112 (Psychology Press, Hove/ New York, 2005)

74. Allison, T., Puce, A. \& McCarthy, G. Social perception from visual cues: role of the STS region. Trends Cogn. Sci. 4, 267-278 (2000)

75. Puce, A. \& Perrett, D. Electrophysiology and brain imaging of biological motion. Philos. Trans. R. Soc. Lond. B Biol. Sci. 358, 435-445 (2003).

76. Miyhashita, Y. Neuronal correlate of visual associative long-term memory in the primate temporal cortex. Nature 335, 817-820 (1988)

77. Sakay, K. \& Miyhashita, Y. Neural organization for long-term memory of paired associates. Nature 354, 152-155 (1991).

78. Cattaneo, L., Sandrini, M. \& Schwarzbach, J. State-dependent TMS reveals a hierarchical representation of observed acts in the temporal, parietal and premotor cortices. Cereb. Cortex 4 Jan 2010 (doi: 10.1093/cercor/bhp291). This study, based on a TMS adaptation paradigm, shows that, although the parietal and frontal motor areas generalize from one effector to another, the STS adapts only when the observed actions are perfomed with the same effector.

79. Silvanto, J., Muggleton, N. \& Walsh, V. State dependency in brain stimulation studies of perception and cognition. Trends Cogn. Sci. 12 , 447-454 (2008).

80. Wood, J. N. \& Hauser, M. D. Action comprehension in non-human primates: motor simulation or inferential reasoning? Trends Cogn. Sci. 12, 461-465 (2008).

81. Buccino, G. et al. Neural circuits involved in the recognition of actions performed by nonconspecifics: an fMRI study. J. Cogn. Neurosci. 16, 114-126 (2004).

82. Bonini, L. et al. Ventral premotor and inferior parietal cortices make distinct contribution to action organization and intention understanding. Cereb. Cortex 5 Oct 2009 (doi: 10.1093/cercor/bhp200).

83. lacoboni, M. et al. Grasping the intentions of others with one's own mirror neuron system. PLoS Biol. 3 529-535 (2005).
84. Hamilton, A. F. C. \& Grafton, S. T. Action outcomes are represented in human inferior frontoparietal cortex. Cereb. Cortex 18, 1160-1168 (2008).

85. Cattaneo, L. et al. Impairment of actions chains in autism and its possible role in intention understanding. Proc. Natl Acad. Sci. USA 104, 17825-17830 (2007)

A fundamental study that shows the presence of motor chains in humans and how their impairment may account for the deficit in intention understanding in children with autism.

86. Deecke, L., Scheid, P. \& Kornhuber, H. H. Distribution of readiness potential, pre-motion positivity and motor potential of the human cerebral cortex preceding voluntary finger movement. Exp. Brain Res. 7, 158-168 (1969).

87. Kilner, J. M., Vargas, C., Duval, S., Blakemore, S.-J. \& Sirigu, A. Motor activation prior to observation of a predicted movement. Nature Neurosci. 7 1299-1301(2004).

88. Jellema, T. \& Perrett, D. I. Cells in monkey STS responsive to articulated body motions and consequent static posture: a case of implied motorion? Neuropsychologia 41, 1728-1737 (2003).

89. Rizzolatti, G. \& Sinigaglia, C. Mirror neurons and motor intentionality. Funct. Neurol. 22, 205-210 (2007).

90. Gallese, V. Before and below theory of mind: embodied simulation and the neural correlates of social cognition. Philos. Trans. R. Soc. Lond. B Biol. Sci. 362, 659-669 (2007).

91. Sinigaglia, C. Mirror in action. J. Conscious. Stud. 16, 309-334 (2009).

92. de Lange, F. P., Spronk, M., Willems, R. M., Toni, I. \& Bekkering, H. Complementary systems for understanding action intentions. Curr. Biol. 18, 454-457 (2008)

93. Brass, M., Schmitt, R. M., Spengler, S. \& Gergely, G. Investigating action understanding: inferential processes versus action simulation Curr Biol. 17. 2117-2121 (2007).

94. Liepelt, R., Von Cramon, D. Y. \& Brass, M. How do we infer other's goals from non stereotypic actions? The outcome of context-sensitive inferential processing in right inferior parietal and posterior temporal cortex. Neuroimage 43, 784-792 (2008).

95. Kilner, J. M. \& Frith, C. Action observation: inferring intentions without mirror neurons. Curr. Biol. 18, R32-R33 (2007)

96. Keysers, C. \& Gazzola, V. Integration simulation and theory of mind: from self to social cognition. Trends Cogn. Sci. 11, 194-196 (2007).

97. Shepherd, S. V., Klein, J. T., Deaner, R. O. \& Platt, M. L. Mirroring of attention by neurons in macaque parietal cortex. Proc. Natl Acad. Sci. USA 106, 9489-9494 (2009).

98. Ishida, H., Nakajima, K., Inase, M. \& Murata, A Shared mapping of own and others' bodies in visuotactile bimodal area of monkey parietal cortex. J. Cogn. Neurosci. 22, 83-96 (2009). An elegant study showing the involvement of mirroring in the recognition of the peripersonal space of others.

99. Colby, C. L., Duhamel, J.-R. \& Goldberg, M. E. Ventral intraparietal area of the macaque: anatomic location and visual response properties. J. Neurophysiol. 69, 902-914 (1993).

100. Duhamel, J.-R., Colby, C. L. \& Goldberg, M. E. Ventral intraparietal area of the macaque: congruent visual and somatic response properties. J. Neurophysiol. 79, 126-136 (1998).

101. Fogassi, L. et al. Coding of peripersonal space in inferior premotor cortex (area F4). J. Neurophysiol. 76, 141-157 (1996).

102. Gentilucci, M., Scandolara, C., Pigarev, I. N. \& Rizzolatti, G. Visual responses in the postarcuate cortex (area 6) of the monkey that are independent of eye position. Exp. Brain Res. 50, 464-468 (1983).

103. Graziano, M. S., Yap, G. S. \& Gross, G. Coding the visual space by premotor neurons. Science 266, 1054-1057 (1994).

104. Godschalk, M., Lemon, R. N., Nijs, H. G. T. \& Kuipers, H. G. J. M. Behaviour of neurons in monkey peri-arcuate and precentral cortex before and during visually guided arm and hand movements. Exp. Brain Res. 44, 113-116 (1981).

105. Hickok, G. Eight problems for the mirror neuron theory of action understanding in monkeys and humans. J. Cogn. Neurosci. 21, 1229-1243 (2009).

106. Catmur, C., Walsh, V. \& Heyes, C. Sensorimotor learning configures the human mirror system. Curr. Biol. 17, 1527-1531 (2007).
107. Heilman, K. M. \& Valenstein, E. Clinical Neuropsychology (Oxford University Press, New York, 2003)

108. Pazzaglia, M., Pizzamiglio, L., Pes, E. \& Aglioti, S. M. The sound of actions in apraxia. Curr. Biol. 18, 1766-1772 (2008)

An important study showing a clear correlation between impairments in action production and action recognition in patients with apraxia.

109. American Psychiatric Association. Diagnostic and Statistical Manual of Mental Disorders 4th edn (American Psychiatric Publishing, Washington, DC, 1994

110. Frith, U. Autism. Explaining the Enigma 2nd edn (Blackwell, Oxford, 2003)

111. Nishitani, N., Avikainen, S. \& Hari, R. Abnormal imitation-related cortical activation sequences in Asperger's syndrome. Ann. Neurol. 55, 558-562 (2004).

112. Bernier, R., Dawson, G., Webb, S. \& Murias, M. EEG mu rhythm imitation impairments in individuals with autism spectrum disorder. Brain. Cogn. 64, 228-237 (2007)

113. Oberman, L. M., Ramachandran, V. S. \& Pineda, J. A Modulation of mu suppression in children with autism spectrum disorders in response to familiar or unfamiliar stimuli: the mirror neuron hypothesis. Neuropsychologia 46, 1558-1565 (2008).

114. Martineau, J., Cochin, S., Magne, R. \& Barthelemy, C. Impaired cortical activation in autistic children: is the mirror neuron system involved? Int. J. Psychophysiol. 68, 35-40 (2008)

115. Théoret, H. et al. Impaired motor facilitation during action observation in individuals with autism spectrum disorder. Curr. Biol. 15, R84-R85 (2005).

116. Dapretto, M. et al. Understanding emotions in others: mirror neuron dysfunction in children with autism spectrum disorders. Nature Neurosci. 9, 28-30 (2006).

117. Avikainen, S., Kulomäki, T. \& Hari, R. Normal movement reading in Asperger subjects. Neuroreport 10, 3467-3470 (1999).

118. Williams, J. H., Whiten, A., Suddendorf, T. \& Perrett, D. I. Imitation, mirror neurons and autism. Neurosci. Biobehav. Rev. 25, 287-295 (2001).

119. Gallese, V. Intentional attunement: a neurophysiological perspective on social cognition and its disruption in autism. Brain Res. Cogn. Brain Res. 1079, 15-24 (2006).

120. Rizzolatti, G., Fabbri-Destro, M. \& Cattaneo, L. Mirror neurons and their clinical relevance. Nature Clin. Pract. Neurol. 5, 24-34 (2009). This paper summarizes recent experiments on the mirror mechanism with particular attention to its role in clinical syndromes.

121. Hamilton, A. F., Brindley, R. M. \& Frith, U. Imitation and action understanding in autistic spectrum disorders: how valid is the hypothesis of a deficit in the mirror neuron system? Neuropsychologia 45, 1859-1868 (2007).

122. Bird, G., Leighton, J., Press, C. \& Heyes, C. Intact automatic imitation of human and robot actions in autism spectrum disorders. Proc. Biol. Sci. 274, 3027-3031 (2007)

123. Leighton, J., Bird, G., Charman, T. \& Heyes, C. Weak imitative performance is not due to a functional 'mirroring' deficit in adults with autism spectrum disorders. Neuropsychologia 46, 1041-1049 (2008).

124. Southgate, V. \& Hamilton, A. F. Unbroken mirrors: challenging a theory of autism. Trends Cogn. Sci. 12, 225-229 (2008)

125. Boria, S. et al. Intention understanding in autism. PIOS ONE 4, e5596 (2009).

Acknowledgements

The authors' work described in this Review was supported by a grant (FIL) of the University of Parma and by grants from Fondazione Monte Parma to G.R. and from Fondazione San Paolo to G.R and C.S. C.S. was also supported by the Italian Ministero dell'Università e della Ricerca, Prin 2007.

Competing interests statement

The authors declare no competing financial interests.

FURTHER INFORMATION

Giacomo Rizzolatti's homepage:

http://www.unipr.it/arpa/mirror/english/staff/rizzolat.htm

ALL LINKS ARE ACTIVE IN THE ONLINE PDF 